\title{
SENSADO INALÁMBRICO DE GASES EN MINERÍA CON SERVICIO WEB EN TIEMPO REAL
}

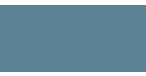

\author{
(Wireless sensing of gas in mining with web service in real time)
}

PhD. Juan Mauricio Salamanca* Germán Andrés Gutiérrez P. **

Martín Edgar Albarracín C. *** Msc. Eduardo Avendaño Fernández***

***,***Grupo de Investigación en Procesamiento Digital de Señales DSP, Universidad Pedagógica y Tecnológica de Colombia (UPTC), Escuela de Ingeniería Electrónica. juan.salamanca@uptc.edu.co, martin.albarracin@uptc.edu.co, germanandres.gutierrez@uptc.edu.co ****Grupo de Investigación en Telecomunicaciones GINTEL, Universidad Pedagógica y Tecnológica de Colombia (UPTC), Escuela de Ingeniería Electrónica. eduardo.avendaño@uptc.edu.co

(Recibido: 5 de Agosto de 2014 y aceptado 1 de Octubre de 2014)

\begin{abstract}
Resumen:
En este trabajo se plasma el desarrollo e implementación de un sistema inalámbrico y sensado de gases explosivos en minería con servicio web en tiempo real. El sistema está compuesto por módulos configurados en una red de sensores dispuestos en las galerías internas de la mina, cada uno estos módulos está en capacidad de recibir señales provenientes de sensores de metano, dióxido de carbono, nivel de carga de la batería y un sensor auxiliar. Cuenta con un módulo Xbee-pro 900 DigiMesh encargado de la transmisión de los datos que entregan los sensores. Estos datos se envían hacia el módulo más externo, el cual conecta con una tarjeta de desarrollo Beaglebone encargada de la visualización de los datos en una plataforma web realizada en software Python, en la que se almacenan los datos de los sensores con el fin de analizar el comportamiento de los gases en el tiempo. La red se implementa en una topología árbol configurando los módulos como routers y uno como coordinador encargado del control de la red. Los módulos se organizan de manera jerárquica, para transmitir los datos hacia la entrada de la mina. En último término se realiza la configuración de la red para que el sistema entre en modo sleep (inactividad) cuando no se está recibiendo información, de esta forma se aumenta la autonomía de las baterías que alimentan a cada uno de los módulos. En este documento se muestra el diseño, implementación y puesta en funcionamiento de un sistema de monitoreo de gases en minería con servicio web en tiempo real basado en una red de sensores Zigbee.
\end{abstract}

Palabras clave: gases, inalámbrico, minería, monitoreo, servicio web.

\section{INTRODUCCIÓN}

Colombia cuenta con grandes reservas de carbón, las cuales ascienden a 6.747 millones de toneladas, que representan el $53.9 \%$ de las reservas de Sur y Centroamérica. Debido a este gran potencial, esta actividad ocupa lugares de privilegio dentro de la economía colombiana representando el $2.3 \%$ del PIB nacional.

\section{Abstract:}

This paper presents the development and implementation of a wireless system and sensing of explosive gases in mining with web service in real time. The system consists of modules configured in a network of sensors arranged in the inner galleries of the mine; each one of these modules has the ability to receive signals from sensors of methane, carbon dioxide, the charge level of battery and an auxiliary sensor. This card has a module $900 \mathrm{Mhz}$ Xbee pro DigiMesh, responsible for transmitting the sensor data. This data is sent to the external module which connects with a card Beaglebone that displays data on a web platform made in Python software, in which sensor data are stored in order to analyze the behavior of gases in time. The network is implemented in a tree topology configuring modules as routers and one as a coordinator that controls the network. The modules are organized hierarchically in order to transmit the data to the entrance of the mine. Finally, the network configuration is done until the system enters in mode sleep (idle) when it is not receiving information, in this way the consuming power decreased, increasing the autonomy of the batteries. This paper describes the design, implementation and operation of a gas monitoring system in mining with web service in real-time based on a network of Zigbee sensors.
Keywords: gases, wireless, mining, monitoring, web service.
Las regiones con mayor producción de carbón son Boyacá y Cundinamarca, que generan alrededor de veinte mil empleos directos y unos 3.000 empleos indirectos.

La explotación minera se ha convertido en una de las fuentes energéticas de mayor impacto, cobrando mayor importancia en los últimos años con el apoyo del Gobierno,
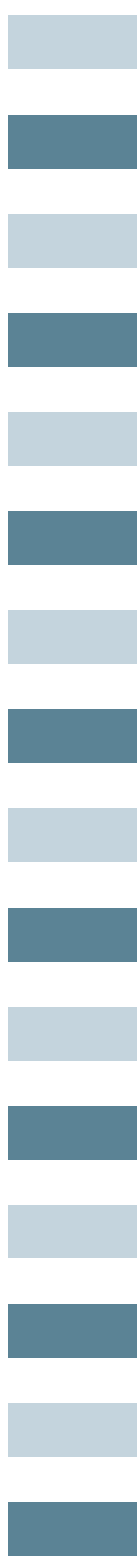
el cual dio origen al Plan Nacional Minero 2007-2010 coordinado por la Agencia Nacional Minera, que ha fomentado la explotación minera como una actividad lucrativa enfocada al aumento de la exportaciones. Pese a los esfuerzos de estas entidades siguen siendo visibles factores como la falta de seguridad industrial y salud ocupacional para los mineros (Echeverri, Cano \& Builes, 2012).

Una de las preocupaciones más grandes para la industria minera es garantizar la preservación de vidas humanas, sin embargo las difíciles condiciones de seguridad industrial dentro del sector minero se ven reflejadas en los altos índices de accidentalidad y riesgos que afectan directamente a los trabajadores, esto se debe en gran parte a la situación inestable y cambiante que se presentan dentro de las minas, estos ambientes se convierten en lugares propicios para la formación de atmósferas explosivas como un detonante invisible y silencioso para el cual existen limitados mecanismos de detección.

En la actualidad, el $95 \%$ de las minas subterráneas de carbón en Colombia no cuentan con un sistema de detección de gases en tiempo real. La detección de gases explosivos se realiza de forma manual, el operario es el encargado de recorrer la mina y alertar de los niveles de estos gases; sin embargo estos métodos tradicionales de medición otorgan una barrera para el análisis de ocurrencia de riesgos de accidentes como contaminación, envenenamiento, sofocamientos o explosiones por concentraciones excesivas de estos gases. Los esfuerzos tecnológicos se centran en la búsqueda de soluciones autónomas para el sensado y alerta de estos gases, una visión interesante es el manejo de información por red de sensores inalámbrico (WSN, wireless sensor network) basada en el protocolo Zigbee (Echeverri et al., 2012).

Una de las aplicaciones hechas en el campo de las redes WSN es el desarrollado en Li, Song, Chen y Ma (2011), donde se utiliza la detección múltiple de gases explosivos, procesando y analizando los cambios que se toman de la muestra en tiempo real. En el desarrollo del proyecto se concluyó que el prototipo no solo funcionaba como elemento didáctico, sino que podrá llegar a ser rentable y efectivo al aplicarlo en entornos como el de la minería. Otro dispositivo que merece la pena resaltar es el desarrollado en la India (Bhattacharyya, Sen, Mandal \& Lahiri, 2006), el cual implementa un sistema para la detección de gases tales como metano y monóxido de carbono, mediante la aplicación de redes WSN. El sensado se hace en forma secuencial, se realiza un sistema de conmutación que permite el intercambio de la instrumentación del sensor, cuando el sensor toma una muestra del gas, este envía una señal modulada en frecuencia que es directamente proporcional al nivel de toxicidad del gas.
Las redes de sensores constituyen una solución para el monitoreo continuo de la acumulación de gases, al implementar esta tecnología en la industria minera los accidentes podrían ser prevenidos, considerando que la comunicación debe ser rápida y eficiente, porque la vida de una persona dentro de una mina $u$ otro campo confinado se puede afectar en cuestión de segundos, debido a la reducción del oxígeno o la presencia de metano, que al contacto con chispa eléctrica puede generar una explosión.

En Colombia, las labores subterráneas se rigen bajo el Decreto 1335 de 1987, que establece las velocidades mínimas para el aire que puede circular en el interior de las minas, así como los valores límites permisibles para los gases que se generan en la explotación. En el artículo $26 \mathrm{se}$ establecen los niveles de gases permitidos para el trabajo en una mina, dentro de los que se encuentran: metano $\mathrm{CH} 4$, dióxido de carbono $\mathrm{CO} 2$, monóxido de carbono $\mathrm{CO}$, ácido sulfhídrico $\mathrm{H} 2 \mathrm{~S}$, anhídrido sulfuroso $\mathrm{SO} 2$, óxido nítrico $\mathrm{NO}$ y dióxido de nitrógeno, además establece que ningún lugar de trabajo bajo tierra debe ser considerado apropiado para trabajar o para pasar por él si su atmósfera contiene menos del $19 \%$ de volumen de oxígeno (Quiceno \& Escobar, 2011).

El artículo está organizado de la siguiente forma: en el capítulo II se da una descripción de red Zigbee, se estudian las topologías y las configuraciones para la implementación en una red de sensores. En el capítulo III se describe el diseño del sistema de monitoreo, se inicia con la implementación de los módulos de sensado y la estación de monitoreo en un servicio web. En el capítulo IV se realiza la descripción del funcionamiento en campo del sistema analizando las variables que intervienen al realizar el sensado de gases en ambientes bajo tierra, finalmente se presentan las conclusiones del trabajo mostrado en artículo.

\section{CARACTERIZACIÓN DE LARED ZIGBEE}

Zigbee es un protocolo inalámbrico de comunicaciones basado en el estándar IEEE 802.15.4 de redes inalámbricas de área personal (WPAN, Wireless Personal Area Network), su objetivo es habilitar redes inalámbricas con capacidades de bajo consumo energético y de bajo costo, que funcionen vía radio y de modo bidireccional, de fácil integración entre sus nodos. Esta tecnología utiliza la banda de frecuencias ISM (Industrial Scientific Medical) para uso industrial, científico y medico con frecuencias de $2.4 \mathrm{GHz}$ siendo la más utilizada, ya que es de uso sin licencia. Su velocidad de transmisión es de $250 \mathrm{Kbps}$, con capacidad de operar en redes de gran densidad con una alta confiabilidad, esto se debe a que cada módulo implementado tiene un identificador de red único, a su vez utiliza un protocolo de comunicación multisalto que puede establecer comunicación entre dos nodos, aun cuando estos se encuentran fuera del rango de transmisión, debido a que puede funcionar en diferentes topologías, malla, estrella y 
árbol. Según la funcionalidad que cumple un módulo en una red, los Zigbee se clasifican en coordinador (ZC), Router (ZR) y dispositivo final (ZED). Para mayor información véase Acosta (2006).

\subsection{Topologías Zigbee}

Topología malla: esta topología establece conexión directa entre cualquier par de nodos, el coordinador está determinado por el primer router que forme parte de la red. Debido a esta característica, este tipo de red presenta una mayor fiabilidad en la transmisión de datos, se gana flexibilidad de la red pero la complejidad de la red aumenta, ya que la comunicación entre un par de dispositivos puede estar determinada por diferentes caminos, lo que conlleva al aumento de los cálculos que debe realizar la red.

Topología estrella: esta topología está compuesta por un dispositivo central que funciona como coordinador y varios dispositivos finales. Cada uno de estos dispositivos está conectado a un coordinador. En esta configuración todos los datos de los dispositivos deben pasar por el coordinador central. Uno de los inconvenientes que se tiene con esta red es que la expansión es muy limitada debido al alcance del coordinador de la red.

Topología árbol: se distingue este tipo de topología como un caso específico de la topología malla. Los dispositivos en esta configuración están organizados de una manera jerárquica, en la cual los routers son los encargados de encaminar los datos, estos están conectados a dispositivos finales, pero estos, a su vez, se pueden comportar como otros routers. Esta topología se puede expandir de forma dinámica como se muestra en Garrido (2009), Ciancetta, Bucci, Fiorucci y Landi (2010).

\subsection{Implementación de la red Zigbee}

Analizadas las topologías y el papel que pueden cumplir los módulos Xbee dentro de una red para una aplicación en minería, se determinó que la configuración más adecuada para la detección de gases, es establecer una topología tipo árbol, ya que esta configuración permite retransmitir los datos de los módulos más alejados. Además, esta configuración permite organizar los módulos de tal manera que la transmisión de los datos cubra las galerías y el túnel principal, con el fin de enviar los datos de los sensores al centro de monitoreo. La topología consta de módulos distribuidos en toda la mina, configurados como routers para establecer un sistema seguro en la transmisión de los datos.

Esta configuración permite que los módulos realicen un escaneo inicial para determinar la ruta de envío de los datos. Sin embargo, si un módulo falla, el sistema está en capacidad de establecer una nueva ruta con los módulos disponibles que estén al alcance de la transmisión.
Al final de los routers se tiene un dispositivo configurado como coordinador encargado de recibir los datos de toda la red, y enlazarlos con la tarjeta de desarrollo BeagleBone. Esta tarjeta realiza la interfaz con una plataforma web donde se visualizan los niveles de gases transmitidos por cada uno de los módulos. La representación gráfica de la red propuesta se muestra en la Figura 1.

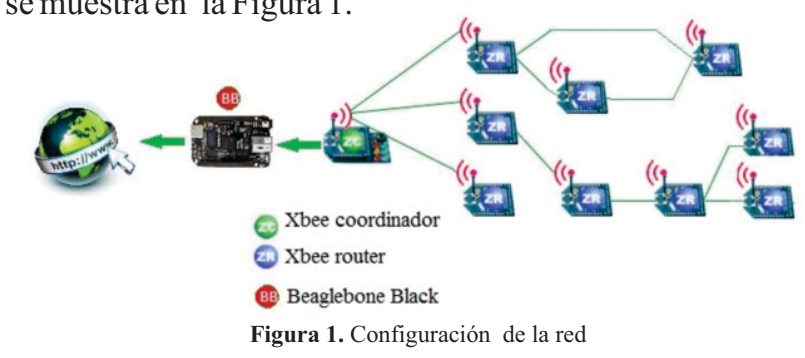

\subsection{Modo sleep de lared}

En la configuración propuesta para la red de sensores, se determina un coordinador encargado de recibir los datos de los routers, los cuales se encuentran distribuidos en los socavones de la mina. Bajo esta premisa se confirma que la actividad de los módulos esté sincronizada por el coordinador, de esta manera la configuración de la inactividad de los módulos estará coordinada por paquetes de sincronización que envía el coordinador hacia los routers. Cada uno de estos recibe estos paquetes y los mantiene durante el funcionamiento de la red, en estos paquetes se especifica el tiempo en que estén enviando datos (todos los módulos activos), y el tiempo que esté la red en inactividad (modo sleep). Configurando la red en modo sleep se reduce el consumo de energía, lo que conlleva al aumento de la autonomía de las baterías que alimentan los módulos. Esta reducción en el consumo de energía se debe a que los módulos permanecen en actividad por cortos periodos de tiempo y se mantienen la mayor parte del tiempo en estado de inactividad.

\section{DISEÑO DEL SISTEMA}

Para un monitoreo continuo de gases en minería se requieren módulos estáticos que supervisen el comportamiento de los gases en distintos puntos de la mina. Teniendo en cuenta este requerimiento se implementan tarjetas modulares, las cuales se distribuyen en los frentes de trabajo y el túnel principal, con el fin de obtener la información de todos los sensores en un centro de monitoreo, el cual se encuentra ubicado fuera de la mina.

. Allí se instala un módulo coordinador encargado de recibir todos los datos y conectarlos con una tarjeta de desarrollo Beaglebone, encargada del procesamiento y visualización de los datos de todos los sensores. . El módulo está diseñado para conexión de tres sensores externos. Inicialmente se contempla la conexión de un sensor de metano TGS 2611-E, un sensor de dióxido de carbono CDM 4161A y se habilita un socket para conexión de otro sensor, el cual depende de las condiciones en las que se instale el sistema. El módulo está alimentado por una batería YSD 998 con un voltaje de entrada de $12 \mathrm{~V}$.
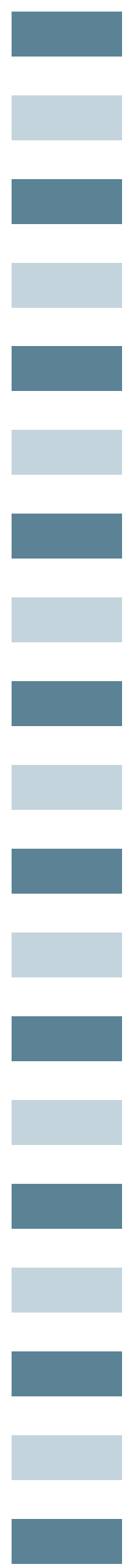
La batería está en capacidad de entregar una corriente de $6500 \mathrm{~mA} / \mathrm{h}$ a descarga lenta. La conexión de la batería con el módulo se realiza mediante un cable Jack a mini-USB.

El diseño de la PCB del módulo se plantea desde dos ópticas distintas: la primera, un sistema autónomo con capacidad de decisión; la segunda, un sistema de conexión en red. Desde estas dos ópticas se distinguen dos elementos principales, un sistema de transmisión inalámbrica Xbee-pro DigiMesh 900 RF y un microcontrolador PIC 16f877a encargado del monitoreo para la activación de alarmas. Por esta razón, las señales provenientes de los sensores y la batería se conectan tanto a los canales de conversión análoga del Xbee como a los del microcontrolador, debido a que el rango de operación de los módulos Xbee está limitado hasta $3.3 \mathrm{~V}$, se realiza una interfaz para señales provenientes de los sensores y la batería por medio divisores de voltaje.

La elección de los módulos Xbee-Pro 900 Digimesh radica en la flexibilidad que tienen para intercambiar datos en una red, pueden ser configurables como dispositivo final, router o coordinador, soportando topologías como punto a punto, punto a multipunto, estrella, malla y árbol. A pesar de tener unas reducidas dimensiones ( $25 \mathrm{~mm} \times 27 \mathrm{~mm})$, proporciona 6 entradas analógicas, 12 entradas-salidas digitales y dos salidas de PWM (Pulse Width Modulation). Todo encapsulado en un dispositivo en 20 pines. Todo lo anterior se programa mediante el software X-CTU, el cual conecta el módulo Xbee con el PC mediante un conector USB. Los módulos pueden establecer ciclos de sueño coordinados en una red con un consumo de energía mínimo. Además de la transmisión inalámbrica que posee, este tipo de dispositivos cuenta con pines de trasmisión y recepción de datos, opera de la banda ISM de $900 \mathrm{MHZ}$, posee un conector RPSMA para instalación antena externa. Para mayor información sobre datos técnicos consultar Becerra (2013).

Para tener compatibilidad entre los datos del Xbee y los datos del PIC 16F877a, se realiza la programación de este de tal manera que la referencia de los canales de conversión análoga a digital sea de $3.3 \mathrm{~V}$. Se habilitan 4 de estos canales $\mathrm{y}$, dependiendo de la toxicidad que arrojan los sensores, se disponen pines de salida para alarmas. El sistema está diseñado de tal manera que se puedan realizar cambios en el algoritmo programado en el microcontrolador, por lo cual se diseñan facilidades de programación desde un conector con el quemador Pickit 2. El empaquetado que se escoge para el desarrollo de la PCB es el TQFP de montaje superficial, que consta de 44 pines ubicados en forma de cuadrado, la separación entre los pines es de $0.8 \mathrm{~mm}$ y la distancia entre lados de $11.4 \mathrm{~mm}$ con un oscilador externo de $4 \mathrm{MHz}$. Para mayor información sobre datos técnicos consultar Microchip Inc. (2014).

EL diseño de la PCB se realiza en Proteus, haciendo uso de los entornos ISIS para las conexiones entre componentes y ARES para el diseño de la placa.
. La tarjeta tiene la capacidad de conexión de 3 sensores con salida análoga en un rango de $0 \mathrm{~V}$ a $5 \mathrm{~V}$. Posee 2 salidas optoacopladas a $12 \mathrm{~V}$, que se utilizan para la conexión de una alarma sonora y una alarma luminosa, y tiene una salida para conexión con la red eléctrica. Todas estas salidas son habilitadas y deshabilitadas por el microcontrolador. La tarjeta se muestra en la Figura 2.

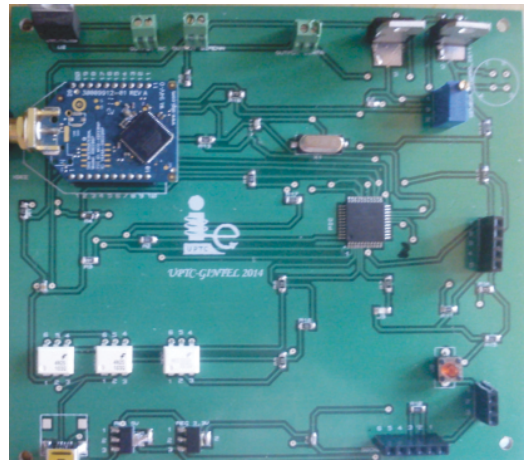

Figura 2. Tarjeta para el sensado inalámbrico de gases en minería

\subsection{Visualización de los niveles de gases}

La visualización de los datos provenientes de los sensores se hace mediante la conexión de un módulo configurado como coordinador de la red, con una tarjeta de desarrollo Beaglebone. En esta se implementa un servidor web, que captura los datos mediante puertos seriales de la tarjeta, y dependiendo de la dirección de origen, se visualizan los niveles de los gases en cada una de las galerías en las que se tengan instalados los módulos.

la identificación de los datos de cada uno de los módulos se realiza mediante la dirección de origen de cada módulo, la cual pertenece a la dirección Mac del dispositivo. Este es el identificador que se le asigna de fábrica a cada uno de los módulos Xbee y que permite reconocerlos dentro de una red. Para el envío de la dirección Mac del módulo a cada uno de estos, se configura en modo API, a fin de que la información que se envía se empaquete en una sola trama. Adicional a esto se configuran los canales de conversión análoga a digital del Xbee, para que la información que entregan los sensores se incluya dentro de la trama. Hechas estas configuraciones, la identificación se lleva a cabo primero por dirección Mac para identificar el modulo y luego se analiza la trama para conocer el valor de cada uno de los sensores. Esto se ejecuta aprovechando la uniformidad de todas las tramas enviadas. En caso de que esta se pierda, se rechaza esta trama y se consideran datos erróneos, y la visualización se mantiene con el último cargado por el servidor.

El diseño del servidor web se hizo en software libre con capacidad de adquirir datos de forma continua, el cual permite monitorear los niveles de gases presentes en las minas subterráneas. El servidor web se desarrolló en Python, este toma muestras y las almacena en archivos de texto plano, para tener un historial de comportamiento de los sensores y niveles de batería en un determinado tiempo. 
En la elección de la plataforma de desarrollo se tuvieron en cuenta aspectos como: integración con el lenguaje de programación Python, bajo consumo de energía, conexión directa a internet, módulos de comunicación seriales, fácil acceso en el mercado, bajo costo. La plataforma seleccionada para el desarrollo del proyecto es una Beaglebone black, esta es una tarjeta de bajo costo con un alto rendimiento y con todas las potencialidades que poseen los sistemas Linux. Dentro de sus principales características se encuentran: disponibilidad de instalar sistemas operativos de distribución gratuita, compatibilidad con gran cantidad de lenguajes de programación, entorno visual configurable, actualizaciones de software disponibles, etc. Además cuenta con un procesador sitara de $1 \mathrm{GHz}$. memoria RAM de $512 \mathrm{Mb}$, lo que lo hace un computador personal muy potente a la hora de ejecutar procesos dispendiosos.

\section{PRUEBAS Y LOGROS ALCANZADOS EN UN AMBIENTE MINERO BAJO TIERRA}

Para determinar el desempeño del sistema se realizaron pruebas dentro de una mina de carbón, en las cuales se evaluaron los siguientes aspectos importantes en el funcionamiento del proyecto: transmisión de datos dentro de ambientes confinados, distancias máximas de transmisión de datos, inconvenientes en la transmisión de datos de RF, pruebas de alarmas, puesta en funcionamiento.

La instalación de la red se hizo de acuerdo con la organización de la topología árbol. En la entrada se instaló un módulo coordinador conectado a la tarjeta de desarrollo Beaglebone, al final del túnel principal se localizó un módulo configurado como router, además se instaló un módulo en una galería de mina, para que realice puente en transición de los datos con el instalado en el túnel principal.

Debido a la robustez de los módulos Xbee pro Digimesh 900 $\mathrm{MHz}$, se logró realizar satisfactoriamente la prueba de transmisión de datos dentro de un ambiente confinado, como lo son las minas, teniendo en cuenta todos los factores que intervienen en el entorno natural de una mina bajo tierra.

La distancia de transmisión que se logró corroborar dentro de la mina es aproximadamente $800 \mathrm{~m}$, con línea de vista que resulta útil para cubrir todo el túnel principal de la mina, lo que da como resultado que los paquetes se entregan de manera eficaz. Cabe resaltar que en este trayecto hay una serie de objetos que podrían llegar a interferir con la señal, como lo son las vagonetas, los ventiladores, estructuras metálicas que sostienen la mina; sin embargo, la prueba fue totalmente satisfactoria al lograr realizar una comunicación entre el emisor y el receptor.

Los mayores inconvenientes que se presentaron en la transmisión de datos fueron aquellos lugares donde se tienen curvas cerradas, ya que la señal se pierde, por esto se hace necesario ubicar módulos repetidores en estos espacios, para que el repetidor tome la señal y la reenvíe hasta el destinatario final.
Se hicieron pruebas de funcionamiento de alarmas, las cuales se instalaron en el módulo y se les aplicó una señal continua de $12 \mathrm{VDC}$, lo que dio como resultado que tanto la alarma sonora como la alarma luminosa son apropiadas, ya que la alarma sonora se escucha en un radio bastante grande debido a que se escogió de 20 w. Con respecto a las alamas luminosas, se diseñaron una serie de flechas en cinta leds, de bajo consumo de energía, las cuales tendrán que guiar al personal por la ruta de evacuación en caso de emergencia.

Para la parte de la prueba en conjunto del sistema, se ubicaron los módulos según la configuración de la red. En el primer módulo se colocó un sensor de dióxido de carbono, sensor de metano, alarma luminosa y una alarma sonora. Para el segundo módulo se ubicó un sensor de metano, un sensor de dióxido de carbono y un potenciómetro que simulaba la otra entrada del sistema, cada módulo fue conectado a la batería de alimentación a una tensión de 12 VDC. Y se evaluaron los resultados.

En primer lugar, el módulo de la galería uno arrojó los resultados esperados. Se le hizo una perturbación al sensor de dióxido de carbono para observar que hubiese los cambios esperados en la página web. El servidor web tomó las señales de forma correcta, ya que se logró observar como cambiaban los datos de la misma forma en que se variaban en el módulo. La perturbación a este sensor se aumentó hasta sobrepasar los umbrales, esto hizo que se activaran las alarmas, tanto luminosa como sonora, las cuales se mantuvieron activas hasta que los niveles disminuyeron. Los valores de los umbrales se pueden variar teniendo en cuenta el tipo de sensor y sus niveles permitidos. Para el módulo de la galería dos, se varió el potenciómetro de manera que no sobrepasara los niveles, en dicho momento el servidor procesó los datos de manera correcta al almacenarlos para la visualización del historial; después, este potenciómetro se movió de tal manera que sobrepasó los umbrales. En ese preciso momento se activó la alarma luminosa, y solo se apagó hasta que descendió el nivel de tensión en el potenciómetro. La implementación y puesta en funcionamiento del sistema en campo se muestra en la Figura 3.

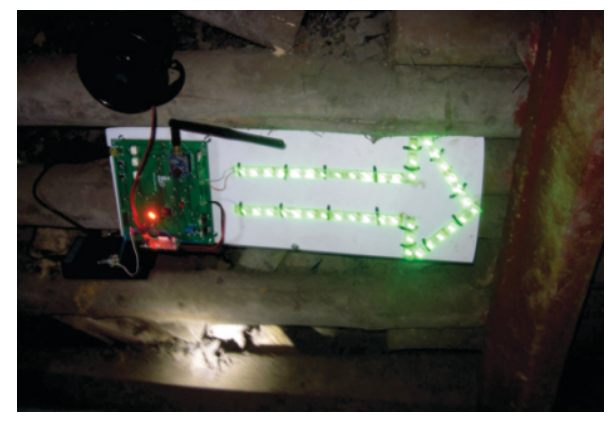

Figura 3. Modulo en operación con alarmas activas

\subsection{Funcionamiento del servicio web}

Se desarrolló un servicio web en software libre, en Python, en el cual se pueden ver tanto los niveles de toxicidad de cada uno de los sensores implementados en la mina, como los niveles de carga de las baterías de cada módulo.
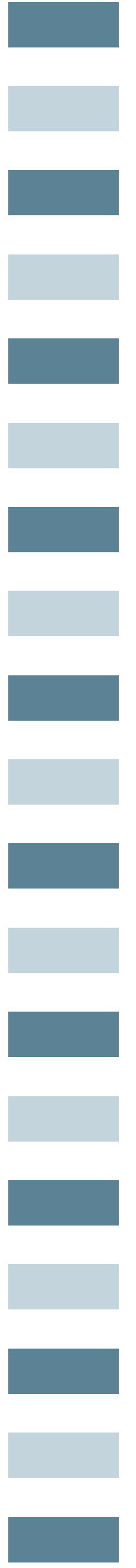
Dentro de los requerimientos para el funcionamiento del servicio web, es necesario que la mina en donde se desee implementar el sistema de sensado de gases, esté provista del servicio de internet, ya sea una red cableada o una red WIFI. Considerando estos dos escenarios, se contemplan las siguientes soluciones: cuando en la mina no exista una conexión directa a internet, esta se hará mediante una conexión directa a la Beaglebone, que estaría alojada en la dirección IP 192.168.7.2, en la cual solo la persona que esté conectada directamente puede llegar a visualizar los datos. $\mathrm{Si}$, por el contrario, en la mina hay servicio de internet, la aplicación se ejecuta en una red LAN, es decir, todo aquel que esté conectado a la red puede llegar a observar los datos. Y, dado el caso en que la mina provea internet y además tenga disponible una red IP pública, la aplicación se ejecutará en dicha red IP, con lo que se podrían ver los datos desde cualquier punto de conexión a internet.

En la Figura 4 se muestra la visualización de los niveles de los gases en una de las galerías de la mina. Estos niveles se representan mediante barras de progreso, las cuales se incrementan o disminuyen de acuerdo con la señal que entregue el sensor dentro de la mina. Adicional a los sensores se muestra otra barra de progreso que indica el nivel de batería del módulo. En este caso, el nivel va excrementando de acuerdo con la descarga de la batería. En todas las barras se muestra la fecha y hora de la muestra que se está visualizando, para tener fiabilidad del dato observado en la plataforma.

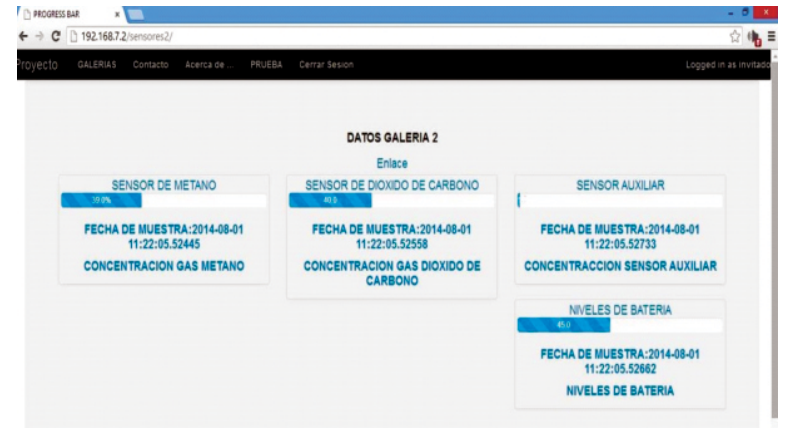

Figura 4. Interfaz web de los datos tomados en una de las galerías de la mina

\section{CONCLUSIONES}

Considerando las pruebas de transmisión de datos de los módulos dentro la mina, es necesario establecer la ubicación precisa de estos para garantizar que la red esté sensando en todos los puntos críticos de la mina, y además, que si un módulo deja de funcionar, el rango de cobertura de este debe ser reemplazado por el más próximo para evitar perder datos de los módulos más interiores.

Se debe tener presente que el nivel de los umbrales para las alarmas de los módulos está sujeto al valor de calibración de los sensores. Por esto es recomendable efectuar calibraciones periódicas, para garantizar que el nivel de los gases esté dentro de los rangos permisibles para la explotación bajo tierra.

\section{REFERENCIAS}

Acosta, M. C. (2006). Estudio del éstandar IEEE 802.15. 4 Zigbee para comunicaciones inalámbricas de área personal de bajo consumo de energía y su comparación en el estándar IEEE 802.15. 1 Bluetooth.

Becerra, E. A. (2013). Diseño e implementación de una red Zigbee mediante módulos Xbee-Z para el monitoreo de sensores de temperatura.

Bhattacharyya, T., Sen, S., Mandal, D. \& Lahiri, S. (2006). Development of a wireless integrated toxic and explosive MEMS based gas sensor. Paper presented at the VLSI Design, 2006. Held jointly with 5th International Conference on Embedded Systems and Design., 19th International Conference on VLSI Design.

Ciancetta, F., Bucci, G., Fiorucci, E. \& Landi, C. (2010). A wireless event-based sensors network for power quality monitoring application. Paper presented at the Power Electronics Electrical Drives Automation and Motion (SPEEDAM), 2010 International Symposium on.

Echeverri, D. R., Cano, S. H. R. \& Builes, J. A. J. (2012). Detección y control de atmósferas explosivas en minas subterráneas de carbón usando programación estructurada. Revista Educación en Ingeniería, 7(14), 10-21.

Li, Y., Song, H. S., Chen, H. X. \& Ma, S. Y. (2011, 10-12 May). Design of automatic system for multiple-gas sensing. Paper presentedat the Instrumentation and Measurement Technology Conference (I2MTC), 2011 IEEE

Microchip. T. Inc.. Pic 16f877a. [Online]. Retrieved from h t t p : / / w w w. m i c r o c h i p. c o m /wwwproducts/Devices.aspx?dDocName $=\mathrm{en} 010$ 242

Quiceno, C. C. C. \& Escobar, J. M. M. (2011). Caracterización de sistemas de ventilación en mineria subterránea. Boletín de Ciencias de la Tierra (29), 73-85 